Cite this article: lonescu-Lungu AV, lacob M, Zetu IN.

Smile Aesthetics

Appreciated by Laypersons.

Stoma Edu J. 2015;2(1):17-24

\section{SMILE AESTHETICS APPRECIATED BY LAYPERSONS}

https://doi.org/10.25241/stomaeduj.2015.2(1).art.2
Andreea-Virginia lonescu-Lungua, Mihnea lacob ${ }^{b^{*}}$, Irina Nicoleta Zetuc

Orthodontic Department, Faculty of Dentistry, ,Gr. T. Popa" University of Medicine and Pharmacy, Jassy, Romania

DMD, Orthodontist DMD, Assistant Professor $\mathrm{DMD}, \mathrm{PhD}$, Associate Professor
Received: 05 September 2014 Accepted: 18 September 2014

\footnotetext{
* Corresponding author:

Assistant Professor Mihnea lacob, DMD, Department of Orthodontics, Faculty of Dentistry "Gr.T.Popa" University of Medicine and Pharmacy, Jassy, Romania 16 Lapusneanu Str, RO-700057, Jassy, Romania Tel/Fax: +40722447659, e-mail: mihneaiacob@yahoo.com
}

\section{Abstract}

Introduction. The new requirements in orthodontics put emphasis on smile aesthetics and yet there are only a few studies on the analysis of smile components and ideal aesthetic smile norms.

Methodology. A lot of 80 pacients has been selected and photos of their smile have been taken. From this photos, 19 pictures have been selected for the smile components they posses and analyzed. There were taken 3 photos for the parallelism of the smile arch with the lower lip; 3 photographs for free margin of the upper lip mucosa; 3 photographs for the anterior height of the smile; 4 photographs for the last maxillary visible tooth in smile; and another 3 photographs for the percentage of the bucal corridor. These photographs have been analyzed by a group of 150 laypersons who have appreciated the esthetics of these photos choosing the most aesthetic and the most unaesthetic of each component.

Results. The parallelism between the smile arch and the inferior lip line, the horizontal linear smile, the medium height of anterior and posterior smile, the last visible maxillary tooth in smile- second premolar and a medium bucal corridor are the most aesthetic smile options. The most unaesthetic variants were considered: a reverse smile arch, a concave smile, a gummy smile, the last visible maxillary tooth in smile- first molar and a large bucal corridor.

Conclusions. The study aim is to establish some dynamic norms for smile aesthetics components.

Keywords: smile, aesthetic, buccal corridor, smile arch, gingival display.

\section{Introduction}

The term "aesthetic" meets beauty, harmony and balance. As you know, beauty and aesthetics are subject to criteria that can vary infinitely, depending on various parameters including age, environment and trends. In recent years, orthodontists, and even the whole population, interest in aesthetics has grown a lot and is still growing (1). Although aesthetics can be very subjective and variable, depending on ethnicity may be defined characteristics of attractive people (2). These characteristics should be taken into consideration during diagnosis and treatment plan whenever you wish to correct a maxillary anomaly. Hassebrauk says that after the eyes, the smile is the second facial feature that people tend to consider when evaluating the attractiveness of a person (3). Facial appearance of a person and its degree of attractiveness have an important impact on social, personal and professional life. The new requirements in orthodontics put emphasis on aesthetic smile and yet there are few studies on the analysis of smile components an ideal aesthetic norms for them.

\section{Methodology}

Our sample consisted of 150 Romanian adults who were recruited by the Department of Orthodontics at the "Gr. T. Popa" University of Medicine and Pharmacy Jassy. They voluntarily completed a questionnaire that included questions about anonymous aesthetic appreciation of the various components 


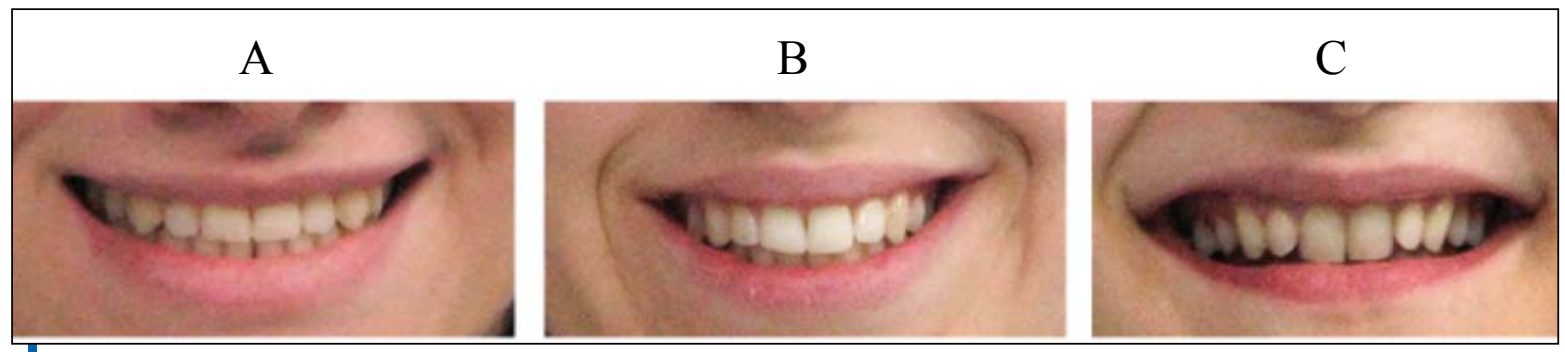

Figure 1. Variants for parallelism of smile arch in relation to the lower lip line

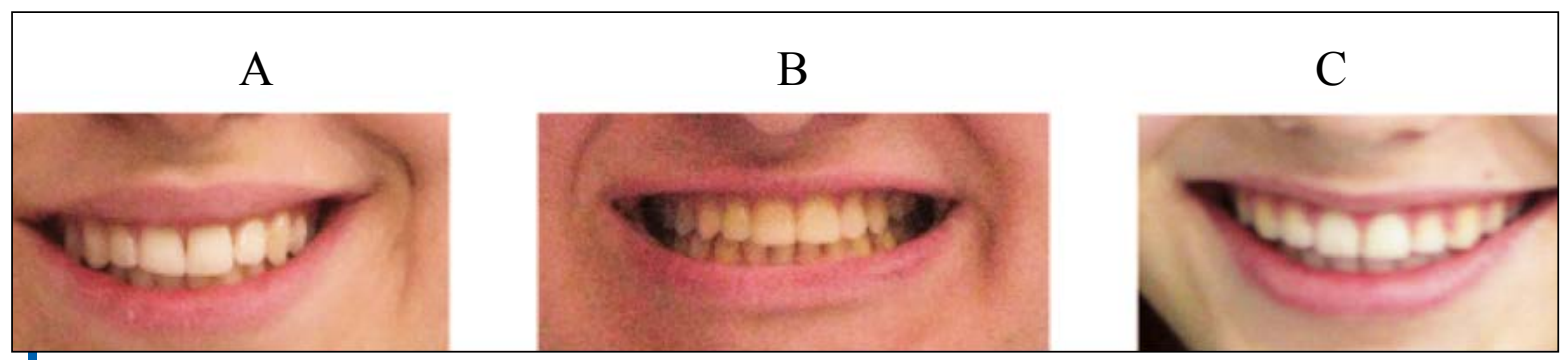

Figure 2. Variants for the free edge of the upper lip mucosa

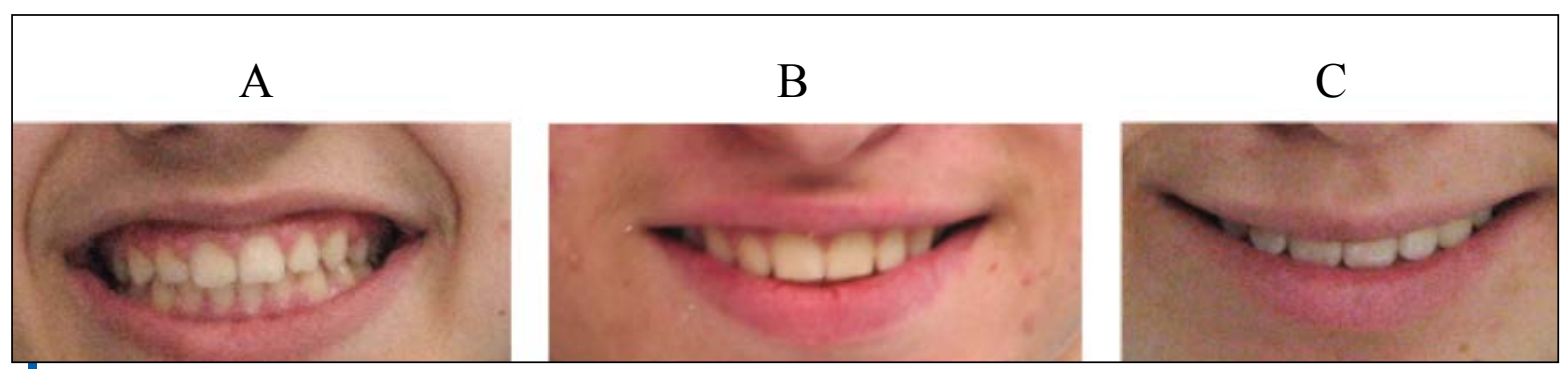

Figure 3. Variants for anterior smile height

of smile. The questionnaire contains 23 questions (12 unique questions, six repeated questions to assess reliability rate) and five questions about demographic information (area of origin, age, gender, ethnicity, completed level of education). Exclusion of individuals in our sample was made only if it worked as dentist or orthodontist, or if it has been treated as an orthodontic patient.

The images presented in the questionnaire were photographs of smile. To obtain them, we selected a group of 80 patients and we took pictures of their smile. Among these pictures were selected 19 images, based on the smile components that have been analyzed. Photos were divided according to aesthetic components that have been analyzed and identified each of them in 6 groups as follows: after parallelism smile arch line relative to the lower lip; free margin of the upper lip mucosa; anterior smile height; posterior smile height; last maxillary tooth visible in the mouth smile and the percentage of buccal corridor.

Parallelism of smile arch in relation to the lower lip line, revealed three variants:

A. parallel smile arch (incisal edges of maxillary canines and premolars cusps, from mesial to distal, follows the curvature of the lower lip);

B. flat (incisal edges of maxillary canines and premolars cusps, from mesial to distal are not presenting a bending upon the lower lip line);

C. reverse (incisal edges of maxillary canines and premolars cusps, from mesial to distal, have a reverse curvature to the lower lip). (Fig. 1)

The free edge of the upper lip mucosa, revealed three variants:

A. horizontal linear smile (free edge of the upper lip mucosa remains horizontal and parallel to cervical tooth line, the plane passing through the commissural points is tangent at median level to red upper lip line);

B. convex (free edge of the upper lip mucosa describes a convex line to the top of the face, commissural plan is passing above the median level of red interior upper lip line);

C. concave (free edge of the upper lip mucosa describes a concave line to the top of the face, commissural plan is passing below the median level of red interior upper lip line) (Fig. 2)

Anterior smile height, has as alternatives:

A. high gingival smile (sees a continuous ribbon of gum above the maxillary central incisor);

B. medium smile (showing $75 \%$ to $100 \%$ of the maxillary central incisors);

C. reduced smile (showing less than $75 \%$ of the upper central incisors ) (Fig. 3) 


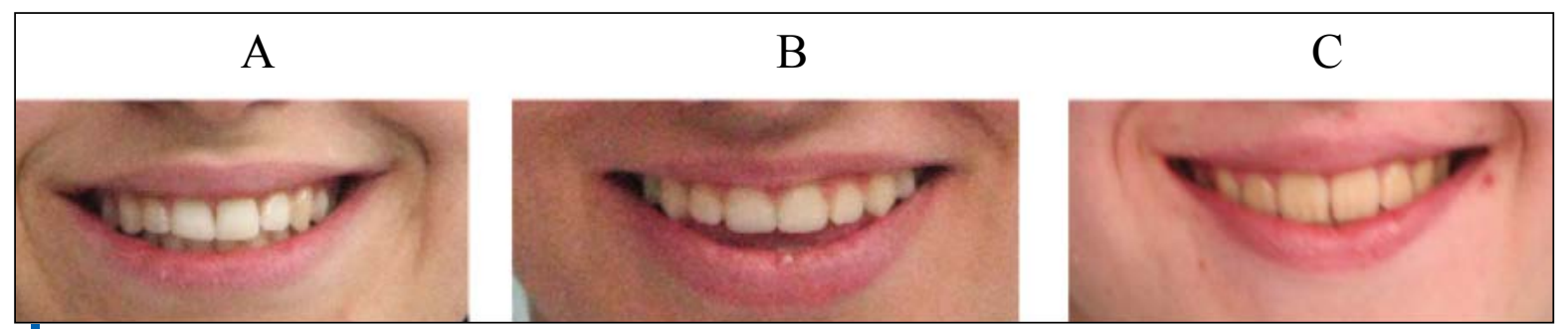

Figure 4. Variants for posterior smile height

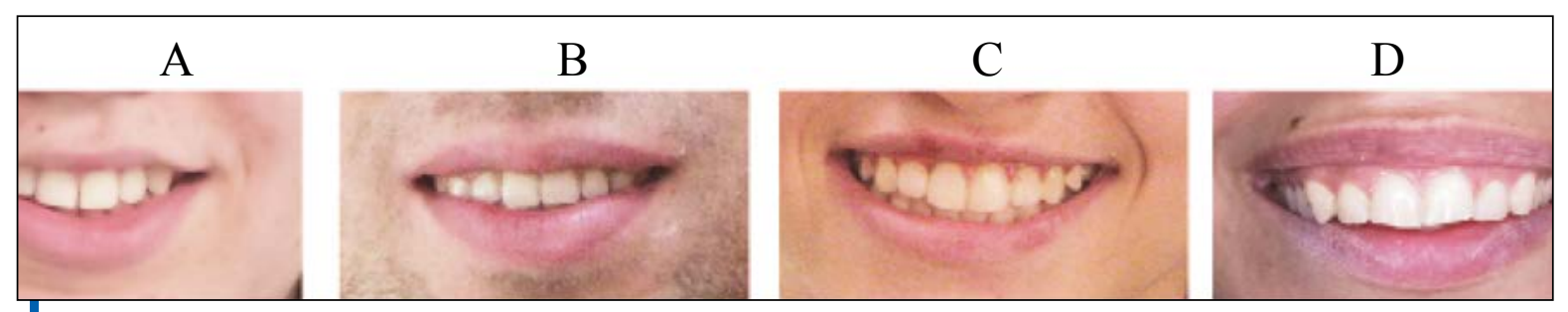

Figure 5. Variants for the last visible maxillary tooth during smile actions

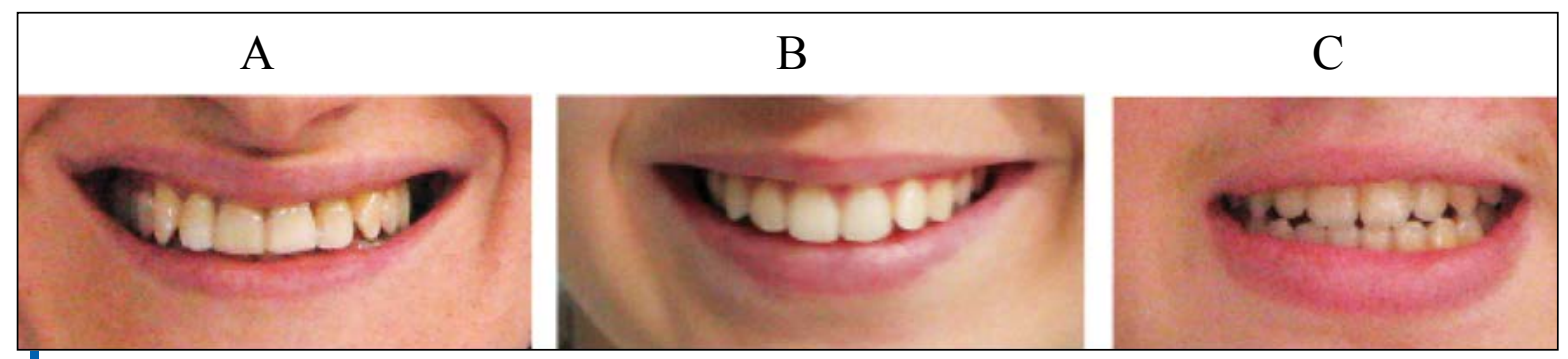

Figure 6. Variants for the percentage of buccal corridor

Posterior smile height, highlights the following variants:

A. high smile (a continuous ribbon of gum above the first maxillary premolar);

B. medium smile (showing $75 \%-100 \%$ of the first maxillary premolar)

C. reduced smile (showing less than $75 \%$ of maxillary first premolar visible). (Fig. 4)

The last visible maxillary tooth during smile actions can be:

the canine;

the first premolar;

the second premolar;

D. the first molar. (Fig. 5)

The percentage of buccal corridor was determined as follows: we measured with a line the distance from the most posterior point of the most posterior tooth on one side at the same point on the contralateral side (maxillary interdental width), and then we measured the distance to the narrowest point visible in the inner corner of the buccal mucosa at the same point on the opposite side. This measurement was divided by the maxillary interdental width. For example, 0.88 means that maxillary dentition occupied $88 \%$ of the intercommisural anterior width and the buccal corridor would be then 12\% (100\% - 88\%) of full smile. This number was recorded and highlighted in three ways:
A. wide / large (18\%);

B. medium (12\%);

C. reduced / small (2\%). (Fig. 6)

These images were analyzed by the group of 150 young people who appreciated the aesthetics of these photos, setting to the most aesthetic and most unsightly of each component.

\section{Results}

The majority of young people (71.33\%) determined for component 1 as the most aesthetic the alternative with the parallelism between smile arch and lower lip line (variant A) and 94.66\% of them felt that a reverse arch smile is the most unsightly (variant C). (Fig. 7)

For component 2 (the free edge of the lining of the upper lip) $62.66 \%$ considered the as the most aesthetic, the horizontal linear smile (version A) and $69.33 \%$ of them considered concave smile as the ugliest (variant B). (Fig 8)

For anterior smile height, $85.33 \%$ appreciated the medium smile as the most aesthetic one (variant B) and tall gum smile was rated as the ugliest (64.66\% - variant A). (Fig. 9)

For posterior smile height, $50.66 \%$ appreciated medium smile as the most aesthetic (variant B), and $42 \%$ rated the tall smile as the ugliest (variant A). (Fig. 10) 


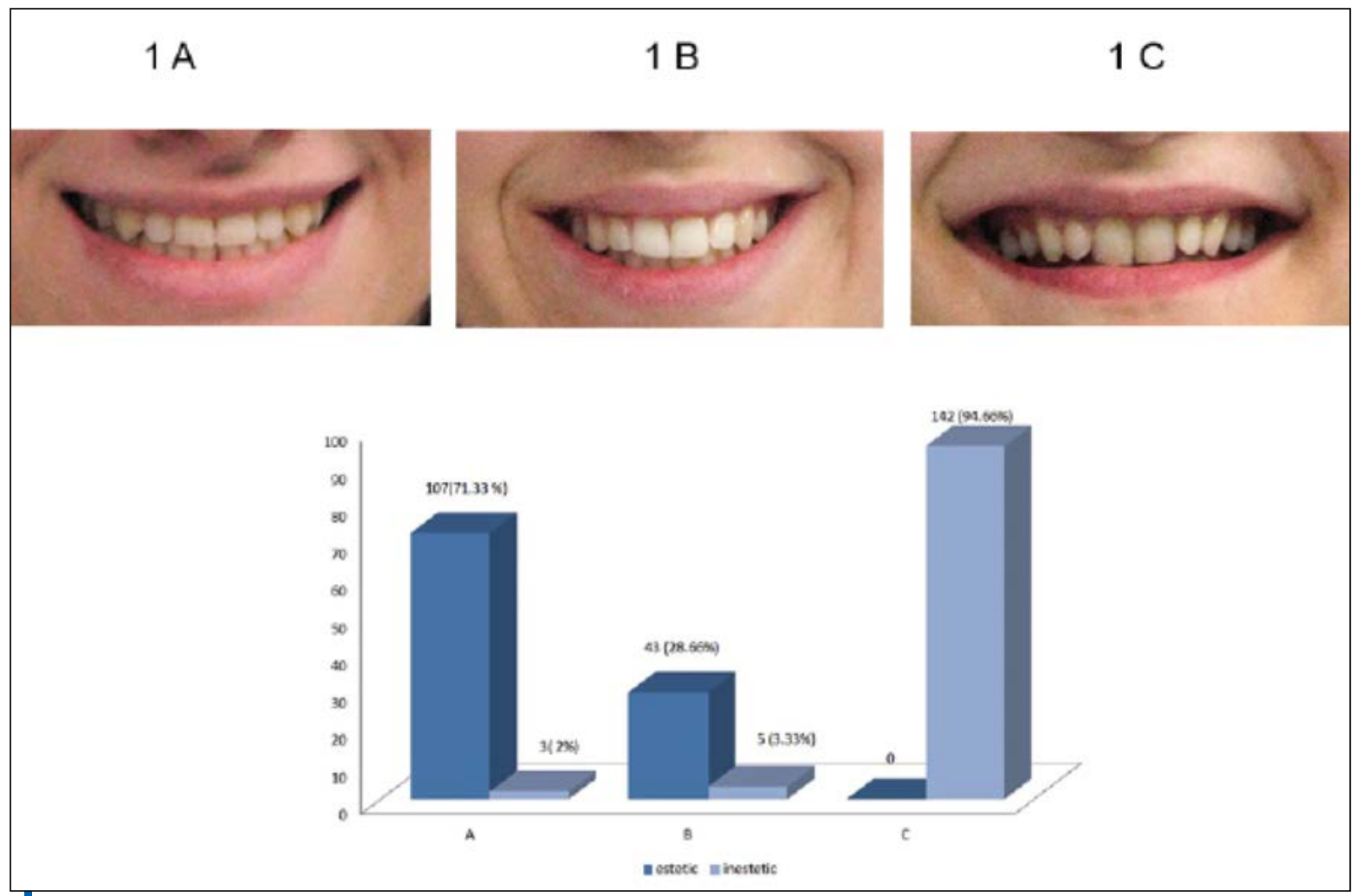

Figure 7. The aesthetic and unaesthetic percentage for each variant of the component 1 , appreciated by the study participants

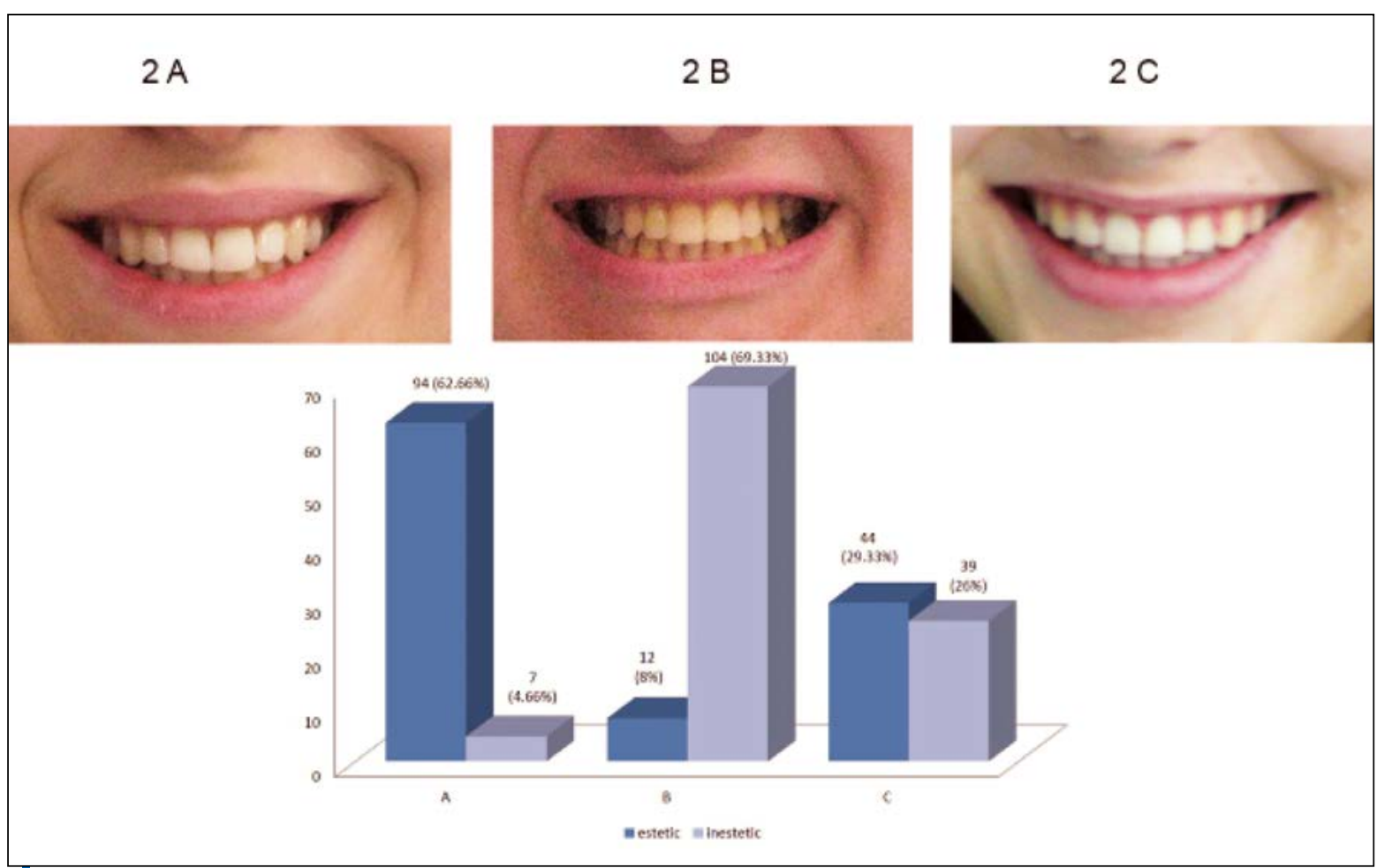

Figure 8. The aesthetic and unaesthetic percentage for each variant of the component 2 ,

appreciated by the study participants

For the last maxillary tooth visible during smile actions, $37.33 \%$ chose the second premolar as the most aesthetic (Variant C) and $56 \%$ rated as the most unsightly the first molar (Variant D). (Fig. 11)

For the last component, $84 \%$ of participants rated an average buccal corridor as the most aesthetic (variant $\mathrm{B}$ ) and $95.33 \%$ rated a wide buccal corridor as the ugliest (variant A). (Fig. 12)

\section{Discussion}

Many studies have been made regarding the facial aesthetics and the aesthetic smile. Some authors 


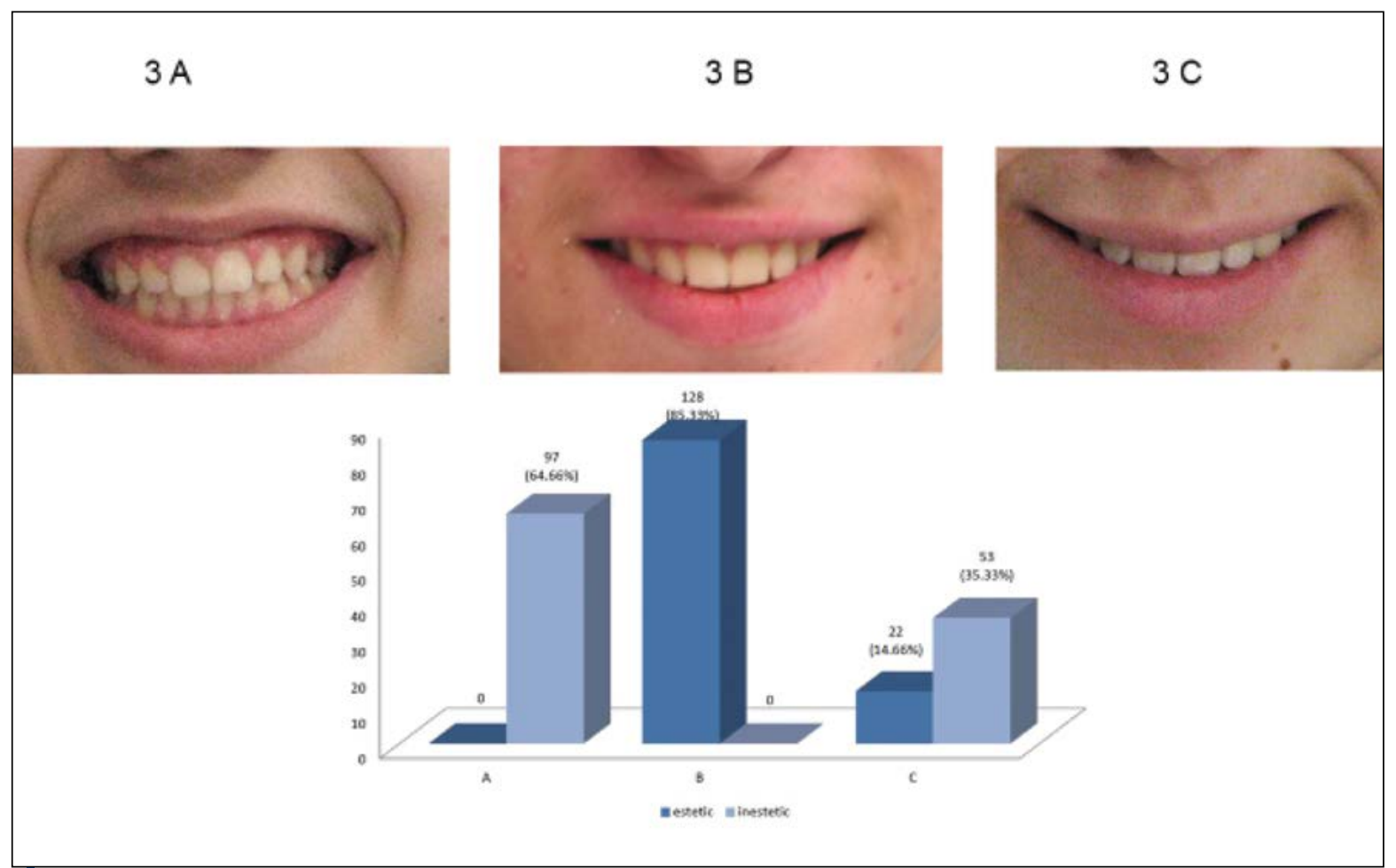

Figure 9. The aesthetic and unaesthetic percentage for each variant of the component 3, appreciated by the study participants

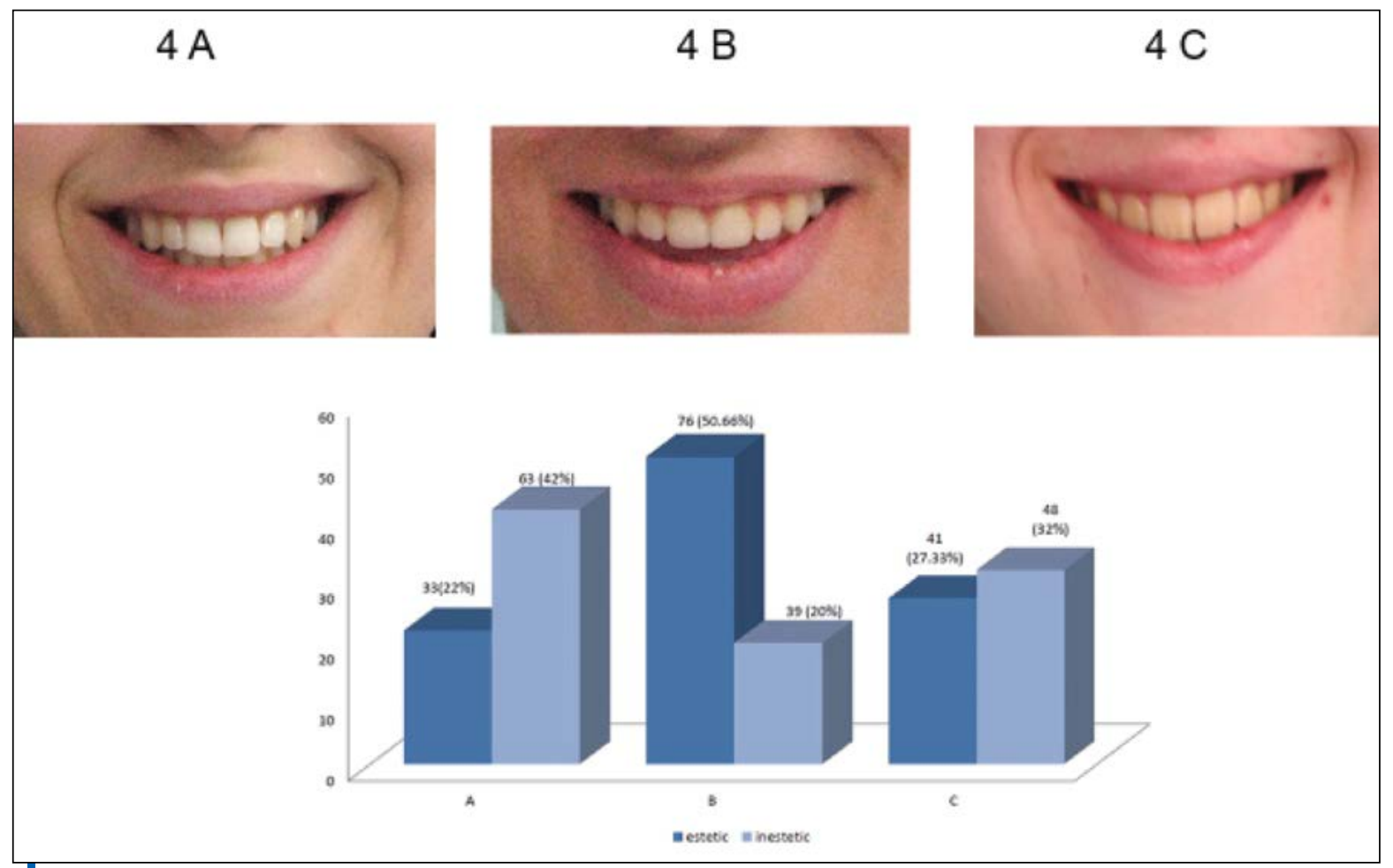

Figure 10. The aesthetic and unaesthetic percentage for each variant of the component 4, appreciated by the study participants

made studies about the cultural differences that exist related to smile characteristics. Clinically significant differences in the preference of smile characteristics were found between Canadian and US laypersons. Canadian laypersons, on average, were more discriminating to deviations from ideal norm and had a narrower range of acceptability. (2)

An other clinician proposes to use digital video and computer technology. In this case the clinician can evaluate the patient's dynamic anterior tooth display and incorporate smile analysis into routine treatment planning. 


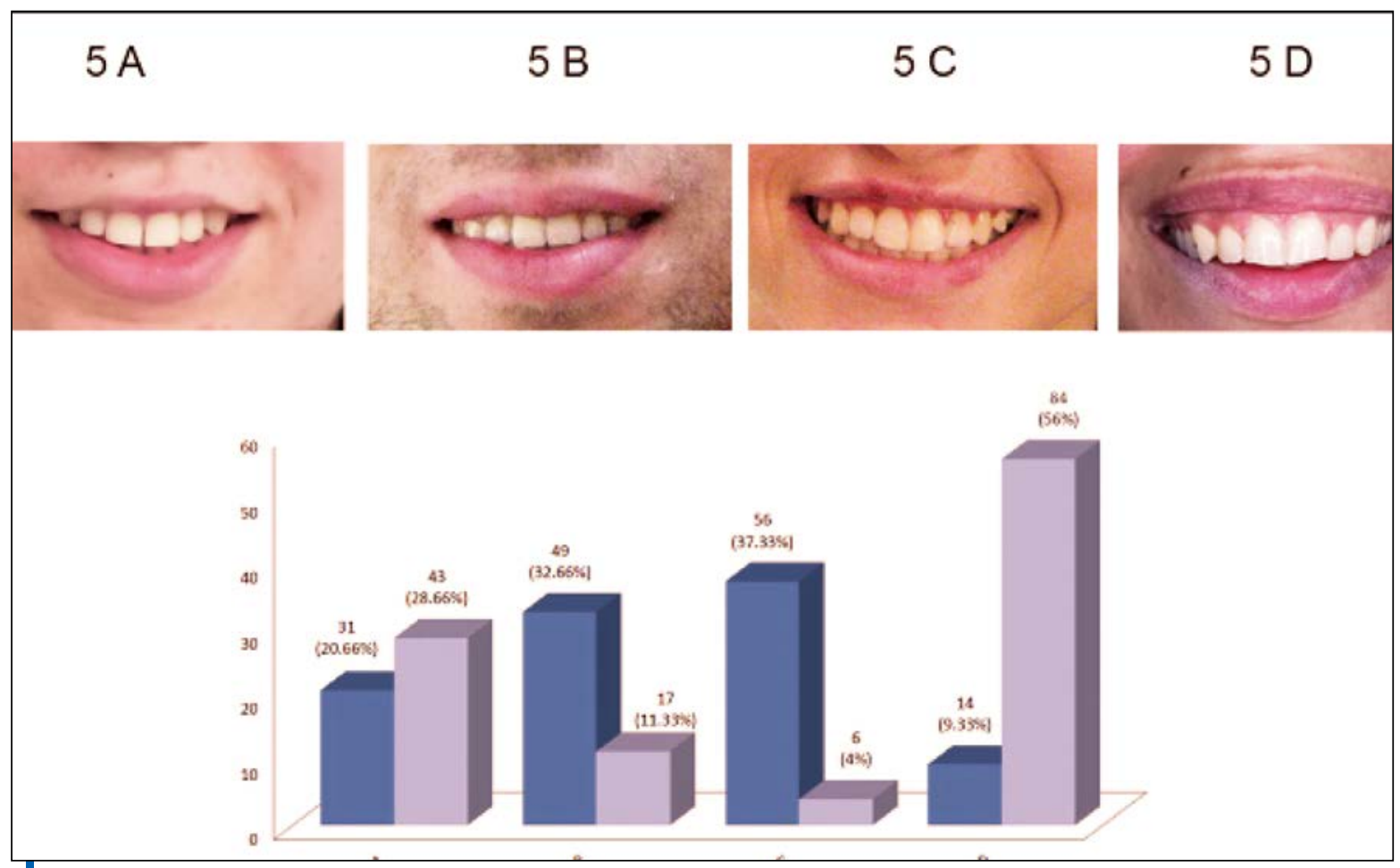

Figure 11. The aesthetic and unaesthetic percentage for each variant of the component 5 , appreciated by the study participants

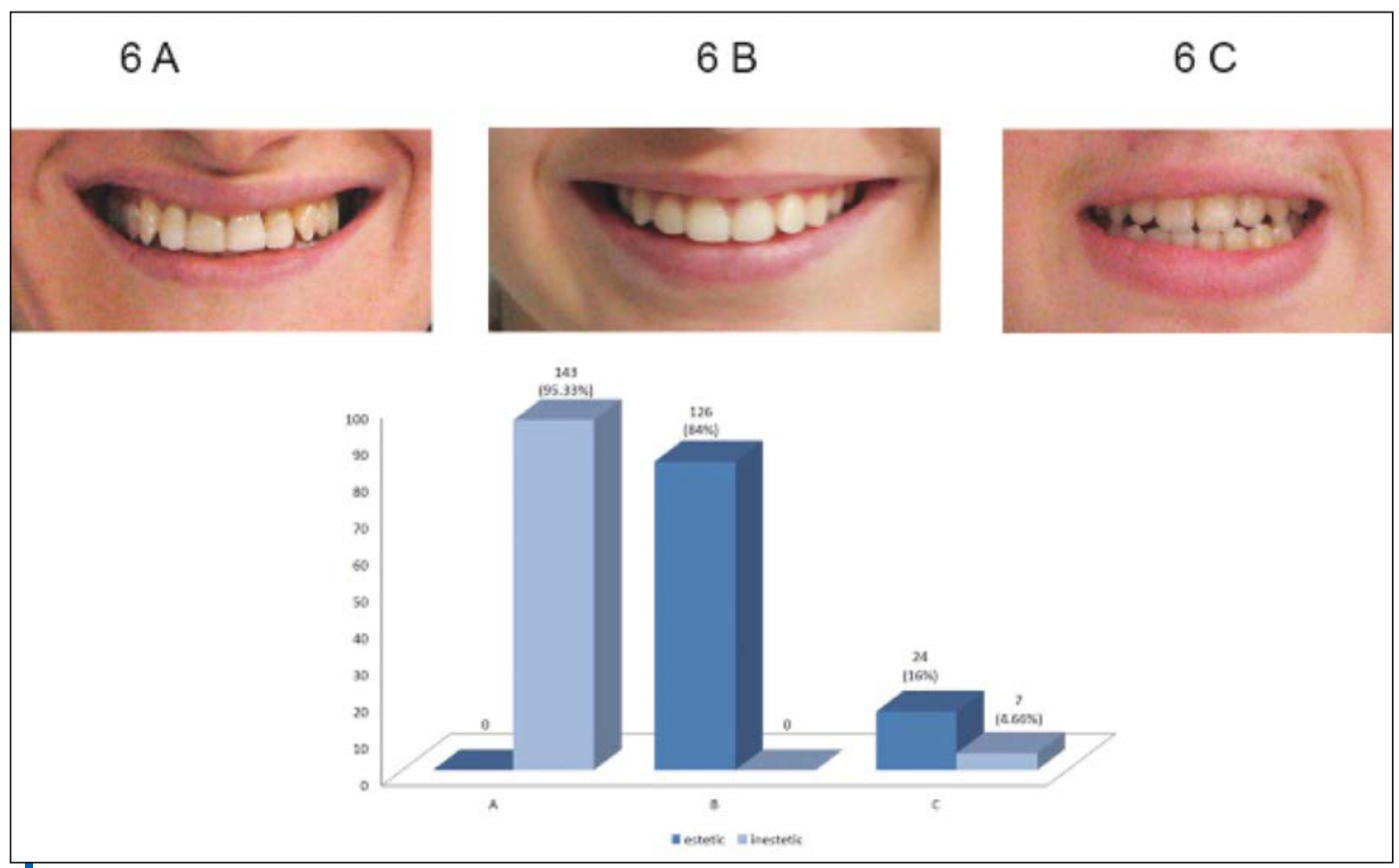

Figure 12. The aesthetic and unaesthetic percentage for each variant of the component 6 , appreciated by the study participants

Aesthetic smile design is a multifactorial decision-making process that allows the clinician to treat patients with an individualized, interdisciplinary approach. $(4,5)$ Others studies on facial aesthetics were made appreciating various smiles of patients with and without rapid maxillary expansion (RME) and compared them after. This study shows that orthodontic treatment might not flatten the smile arch as previously suggested, and, furthermore, that RME appears to be associated with a decreased buccal corridor. (6) 
The objective of another study was to evaluate the role of the posed smile in overall facial esthetics, as determined by laypersons and orthodontists. The presence of a malocclusion has a negative impact on facial attractiveness. Orthodontic correction of a malocclusion affects overall facial esthetics positively. Laypeople and orthodontists agree on what is attractive. (7) Other study shows that the orthodontically treated subjects had significantly poorer smile scores than the subjects with "normal occlusion. The height of the upper lip to the upper central incisor influenced the rating of the smile, the most attractive smiles having the upper lip at the height of the gingival margin of the upper central incisor.(8)

Some authors also try to evaluate the aesthetic smile by determinating whether asymmetric and symmetric anterior dental discrepancies are detectable by dental professionals and laypersons. Orthodontists were more critical than dentists and laypeople when evaluating asymmetric crown length discrepancies. A small midline diastema was not rated as unattractive by any group. Unilateral reduction of papillary height was generally rated less attractive than bilateral alteration Asymmetric alterations make teeth more unattractive to not only dental professionals but also the lay public. (9) Other study evaluated the hard-and soft-tissue contributions to the esthetics of the posed smile in growing patients seeking orthodontic treatment. Vertical lip thickness proved to be the most influential variable in smile aesthetics. The significant relationship of incisor protrusion with the vertical thickness of the vermilion border of the upper lip must be considered when planning orthodontic treatment. (10)

\section{Conclusion}

This study helps us to establish a set of dynamic norms for the aesthetics of smile components. Thus, the parallelism of smile arch with the upper lip smile, horizontal linear smile, an anterior and posterior medium smile height, the last visible tooth during smile actions has to be the second premolar and a medium buccal corridor are the most aesthetic variants. The most unsightly variants have been rated the follows: a reverse smile arch of a hollow smile, a concave smile, an anterior and posterior height smile, the last visible tooth during smile actions is the first molar and a smile with a wide buccal corridor. For the success of orthodontic treatment , both in terms of aesthetic, functional and occlusal is very important for the orthodontist to know all the details about the ideal aesthetic norms of smile.(1)

In this paper we wanted to emphasize the importance of aesthetic norms of smile for orthodontic treatment and summarize, if

\section{Bibliography}

1. Zetu I, Pacurar M. Ortodontie. Tehnica arcului drept. Analize necesare, Vol. I,Tg. Mures: Editura Lyra; 2003.

2. McLeod C, Fields HW, Hechter F, Wiltshire W, Rody W Jr, Christensen J. Esthetics and smile characteristics evaluated by laypersons. Angle Orthod. 2011;81(2):198-205.

3. Hassebrauck M. The visual process method: A new method to study physical attractiveness. Evol Hum Behav 1998;19(2):111-123.

4. Ackerman JL, Ackerman MB, Brensinger CM, Landis JR. A morphometric analysis of the posed smile. Clin Orthod Res 1998;1(1):2-11.

5. Ackerman MB, Ackerman JL. Smile analysis and design in the digital era. J Clin Orthod. 2002;36(4):221-236.

6. Maulik C, Nanda R. Dynamic smile analysis in young adults. Am J Orthod Dentofacial Orthop. 2007;132(3):307-315.

7. Havens DC, McNamara JA Jr, Sigler LM, Baccetti $\mathrm{T}$. The role of the posed smile in overall facial esthetics. Angle Orthod. 2010;80(2):322-328.

8. Hulsey CM. An esthetic evaluation of lip-teeth relationships present in the smile. Am J Orthod 1970;57(2):132-144.

9. Kokich VO Jr, Kiyak HA, Shapiro PA. Comparing the perception of dentists and lay people to altered dental esthetics. J Esthet Dent 1999;11(6):311324.

10. McNamara L, McNamara JA Jr, Ackerman MB, Baccetti T. Hard- and soft-tissue contributions to the esthetics of the posed smile in growing patients seeking orthodontic treatment. Am J Orthod Dentofacial Orthop. 2008;133(4):491-499. 


\section{Andreea-Virginia lonescu-Lungu}

DDS, Orthodontist

Department of Orthodontics, Faculty of Dentistry, "Gr. T. Popa”

University of Medicine and Pharmacy, Jassy, Romania

She graduated the Faculty of Dentistry, "Gr.T.Popa" University of Medicine and Pharmacy, Jassy, Romania in 2011. In the same year she became resident in Orthodontics. Now she is an Orthodontist. Doctor lonescu collaborated on a book as co-author together with

Associate Professor Irina Nicoleta Zetu, Chief of Department of

Orthodontics from Jassy. She has participated in many national and international congresses with a series of presentations,

lectures and posters; one of the posters won the 1 st prize at the

international congress Stomis-2012, from Jassy, Romania. She

has participated at over 20 national and international courses of orthodontic professional training. One of her main research interests is aesthetics, especially the dento-facial field.

\section{Questions}

Which is the following is not a variant for the lower lip line?

$\square$ a. Flat smile arch;

b. Parallel smile arch;

c. Flat;

ad. Reverse.

\section{Which is the following is not a variant for the free edge of the upper lip mucosa?}

$\square$ a. Horizontal linear smile;

$\square$ b. Vertical linear smile;

c. Convex;

ad. Concave.

\section{Which is not an alternative for anterior smile height?}

$\square$ a. High gingival smile;

b. Medium smile;

c. Small smile;

$\square \mathrm{d}$. Reduced smile.

\section{Which is not a variant for posterior smile height?}

a. High smile;

$\square$ b. Small smile;

c. Medium smile;

$\square$ d. Reduced smile. 